\title{
Diferenças na gravidade da queda entre idosos jovens e longevos
}

\section{Differences in gravity fall between elderly young and the oldest old}

\author{
Gabriela Guimarães Oliveira', Bruna Borba Neves², Luisa Braga Jorge ${ }^{3}$, Jéssika Cefrin Dantas Neris ${ }^{4}$, \\ Bruna Rios Rauber ${ }^{5}$, Iride Cristofoli Caberlon' ${ }^{6}$ Ângelo José Gonçalves Bós ${ }^{7}$
}

\footnotetext{
' Fisioterapeuta, Mestranda em Gerontologia Biomédica, Instituto de Geriatria e Gerontologia (IGG), Pontifícia Universidade Católica do Rio Grande do Sul (PUCRS), Porto Alegre, RS, Brasil.<oliveira_gabriela@hotmail.com>

2 Terapeuta Ocupacional, Mestranda em Gerontologia Biomédica, Instituto de Geriatria e Gerontologia (IGG), Pontifícia Universidade Católica do Rio Grande do Sul (PUCRS), Porto Alegre, RS, Brasil. <brunanevesto@hotmail.com>

3 Fisioterapeuta, Mestranda em Gerontologia Biomédica, Instituto de Geriatria e Gerontologia (IGG), Pontifícia Universidade Católica do Rio Grande do Sul (PUCRS), Porto Alegre, RS, Brasil. <lbragajorge@hotmail.com>

${ }^{4}$ Enfermeira, Pontifícia Universidade Católica do Rio Grande do Sul (PUCRS), Porto Alegre, RS, Brasil. <jehg3@hotmail.com>

${ }^{5}$ Fisioterapeuta, Pontifícia Universidade Católica do Rio Grande do Sul (PUCRS), Porto Alegre, RS, Brasil.<brurauber@gmail.com>

${ }^{6}$ Enfermeira, Doutora em Gerontologia Biomédica pela Pontifícia Universidade Católica do Rio Grande do Sul (PUCRS). Professora do Curso de Enfermagem da Universidade Luterana do Brasil (ULBRA) Gravataí, RS, Brasil. <iridec27@gmail.com>

Médico Geriatra, PhD pela Universidade de Tokai - Japão. Professor Titular do Instituto de Geriatria e Gerontologia (IGG) e do Programa de Pós-Graduação em Gerontologia Biomédica, Pontifícia Universidade Católica do Rio Grande do Sul (PUCRS), Porto Alegre, RS, Brasil. <angelo.bos@pucrs.br>
}

\section{ARTICLE INFO}

\section{Article history}

Received: $27 / 12 / 2016$

Accepted: 18/03/2017

\section{Correspondent Author}

Gabriela Guimarães Oliveira

Av. Wenceslau Escobar, 1086/B516

90900-000 Porto Alegre, RS, Brasil

<oliveiragabriela@hotmail.com>

(C) 2016 All rights reserved

Editors

Alfredo Cataldo Neto

Paula Engroff

\begin{abstract}
RESUMO
Objetivo: Observar as possíveis diferenças na gravidade da queda entre idosos jovens e longevos. Métodos: Foram identificados todos os idosos jovens (60 a 79 anos) e longevos (80 anos ou mais) atendidos por queda no ano de 2010, em duas unidades de Atendimento de Urgência e Emergência Hospitalar em Porto Alegre, RS. Mediante revisão dos boletins de atendimento, foram extraídos dados referentes ao idoso, dentre eles, as consequências da queda, classificadas pelo índice proposto por Caberlon e Bós (2015). As médias da gravidade da queda foram comparadas entre os dois grupos etários e testada pelo teste † de Student. A relação entre o gênero, faixa etária e o grau de gravidade foi testado pelo Qui-quadrado. Resultados: A maioria dos atendidos era do sexo feminino (72\%), entre as quais $24 \%$ eram longevas. A percentagem de longevas foi significativamente maior que o de longevos (19\%, $p<0,001)$. A média do índice de gravidade geral foi de 5,7. Longevos apresentaram um índice de 6,5 $\pm 5,45$ enquanto os idosos mais jovens apresentaram um índice menor de 5,5 $\pm 6,39(p<0,001)$. As mulheres apresentaram maior frequência de gravidade severa. Conclusão: Concluímos que idosos longevos apresentam maior gravidade das quedas. Essa conclusão também é válida para o sexo feminino que, além de caírem com maior frequência apresentam maior gravidade das quedas principalmente no número de fraturas nas faixas etárias avaliadas (idosos jovens e longevos). Também concluímos ser possível utilizar um instrumento de avaliação da qualidade da queda que poderá ser utilizada em investigações futuras, a fim de contribuir para a realização de uma vigilância epidemiológica mais eficaz e resolutiva. PALAVRAS-ChAVE: Envelhecimento; Idoso; Idoso de 80 Anos ou mais; Acidentes por Quedas; Gravidade do Paciente.
\end{abstract}

\begin{abstract}
Objective: To observe the possible differences in the severity of the decline among elderly young and the oldest old. Methods: We identified all elderly young (60-79 years) and oldest old (80 or older) admitted to fall in 2010, in two Urgent Care Units and Emergency Hospital in Porto Alegre. Upon review of the service bulletins, data were extracted for the elderly, among them, the fall of the consequences, classified by the index proposed by Caberlon and Bos (2015). The mean decrease in severity were compared between the two age groups and tested by Student's $t$ test. The relationship between gender, age and the degree of severity has been tested by chi-square. Results: Most attended were female (72\%), among which $24 \%$ were long-lived. The percentage of long lifetime was significantly higher than the oldest ( $19 \%, p<0.001)$. The overall average severity score was 5.7 . Oldest old showed an index of $6.5 \pm 5.45$ while the elderly young had a lower rate of $5.5 \pm 6.39(p<0.001)$. Women showed a higher frequency of severe gravity. Conclusion: We conclude that the oldest old have higher severity of falls. This conclusion also applies to the female sex, and fall more often have higher severity of falls mainly in the number of fractures in the evaluated age groups (young and the oldest old). Also completed is possible to use an assessment tool of decrease in quality that can be used in future research in order to contribute to the achievement of a surveillance more effective and resolute.

KEYWORDS: Aging; Aged; Aged, 80 and over; Accidental Falls; Patient Acuity.
\end{abstract}




\section{INTRODUÇÃO}

O envelhecimento populacional além de ser um fenômeno mundial mostra-se como um dos principais desafios da atualidade. É consequência do crescimento acelerado da população idosa devido à diminuição das taxas de natalidade e mortalidade e do aumento da expectativa de vida que acaba por gerar mudanças significativas na pirâmide etária ${ }^{1}$.

Atualmente, no Brasil, a população de idosos compreende 18 milhões de pessoas, representando $12 \%$ da população total ${ }^{2}$. Projeções indicam que, nos próximos vinte anos, a população idosa brasileira poderá exceder trinta milhões de pessoas chegando a representar quase $13 \%$ da população do país ${ }^{3}$. Nesse contexto, o Rio Grande do Sul e Porto Alegre são considerados o estado e a capital com maior percentual de idosos, representando, respectivamente, $13,66 \%$ e $15,04 \%$ da população².

Os longevos (80 anos ou mais) correspondem à faixa etária mais crescente no mundo, com um aumento em torno de $81 \%$, de acordo com o censo de 2010. As estimativas indicam que, em 2050, esta faixa etária será de 13,8 milhões de pessoas ${ }^{2,4}$. Assim, atualmente, o Rio Grande do Sul é o quarto estado em número de longevos, com um percentual de 1,88\%. Já Porto Alegre é a capital brasileira com maior número de nonagenários, correspondendo a 4.682 pessoas, e a 18a em número de centenários, correspondendo a 150 pessoas $^{2}$.

Sabe-se que o envelhecimento se dá de maneira diferente e em progressões diferentes, à medida que existem fatores como genética e estilo de vida que também influenciam. Porém, existem alterações durante o processo de envelhecimento a nível celular que acarretam mudanças físicas e declínio em funções orgânicas que se relacionam com o avançar da idade ${ }^{5}$ No sistema musculoesquelético ocorre à diminuição da densidade mineral óssea, força muscular, flexibilidade e resistência. Já as alterações no sistema nervoso provocam uma redução na condução nervosa e lentidão das reações corporais, interferindo na coordenação e propriocepção. $O$ envelhecimento traz consigo também prejuízo em sistemas sensoriais como visão e audição que são de fundamental importância na adaptação ao meio $^{6}$.

Assim, a morbidade é um dos principais indicadores de saúde analisados na população longeva, ao passo que a sua prevalência é maior devido à alta frequência de condições crônicas individuais que acabam por comprometer a capacidade funcional ${ }^{7}$. A capacidade funcional relaciona-se à capacidade que a pessoa possui de viver de maneira autônoma e independente.
A perda desta capacidade, geralmente esta associada a maior risco de complicações, como por exemplo, a queda ${ }^{8}$.

A queda em idosos constitui um dos principais problemas de saúde pública. Em decorrência de sua alta incidência é considerada a principal causa de morbidade, mortalidade e perda de qualidade de vida nessa população9. A queda é um evento acidental que tem como resultado a mudança de posição do indivíduo para um nível mais baixo em relação a sua posição inicial, com incapacidade de correção em tempo hábil e apoio no solo ${ }^{10}$.

Atualmente, imensos são os estudos que avaliam a ocorrência de quedas, no entanto, escassos são aqueles que avaliam sua gravidade. Tal fato, mostra-se preocupante, ao passo que as consequências geradas pelas quedas são inúmeras. Entre elas, as lesões no corpo, à diminuição da mobilidade, com consequente restrição de atividades, a institucionalização e até mesmo a morte. Além de prejuízos físicos elas podem causar danos psicológicos, como o medo de cair, além de aumento dos custos com cuidados de saúde ${ }^{11}$. As quedas provocam em 40 a 60\% das vezes, algum tipo de lesão. Entre as principais lesões destacam-se a presença de escoriações, edemas, equimoses e fraturas ${ }^{12}$.

Entre os idosos que sofreram queda, em um ano dois terços apresentarão nova queda. A prevalência da queda aumenta com a idade, atingindo 50\% da população superior a 80 anos. Assim, quanto mais frágil o idoso, maior o risco de queda e a gravidade do evento ${ }^{13}$.

Desta forma, considerando o aumento da população idosa e a queda como um evento frequente e grave nesta população, este estudo tem por objetivo observar as possíveis diferenças na gravidade da queda entre idosos jovens e longevos.

\section{METODOLOGIA}

Este estudo caracteriza-se por ser transversal, retrospectivo, descritivo-analítico, quantitativo, por revisão de boletins de atendimento de idosos. Foram identificados todos os idosos (60 anos e mais) atendidos por queda no ano de 2010, em duas unidades de Atendimento de Urgência e Emergência Hospitalar (UAUEH) do Sistema Único de Saúde (SUS) na capital do Estado do Rio Grande do Sul, Porto Alegre, sendo elas o Hospital Cristo Redentor e Hospital de Pronto Socorro (HPS). Mediante revisão dos boletins de atendimento, arquivados no Serviço de Arquivo Médico e Estatística (SAME) de cada hospital pesquisado, foram extraídos dados referentes ao idoso, dentre eles, as consequências da queda. A 
idade dos pacientes foi dividida em dois grupos: idosos jovens (60 a 79 anos) e longevos (80 anos ou mais).

Foram levados em conta os seguintes critérios de inclusão: levantamento no Sistema de Registro de todos os boletins de atendimento das instituições pesquisadas, por queda e suas consequências, no ano de 2010; revisão de todos os boletins de atendimento das pessoas idosas (60 anos e mais), cujo motivo de atendimento foi por queda, no período de $1^{\mathrm{o}}$ de janeiro a 31 de dezembro do ano de 2010; indivíduos idosos cuja procedência era somente das regiões Metropolitana de Porto do Estado do Rio Grande do Sul; registro de todos os tipos de quedas e de suas consequências que os idosos sofreram, idosos que caíram no período de primeiro de janeiro a 31 de dezembro e tiveram ou não fratura mediante confirmação pelo resultado da investigação radiográfica; levantamento de todas as quedas e suas consequências, pela revisão individualizada de todos os boletins de atendimento em cada instituição onde constassem as informações como: idade do atendido, sexo, bairro, município, data do atendimento, hora, motivo do atendimento, data da queda (dia, mês, ano, estação do ano), horário e período do dia da queda; local da queda (no domicílio e fora dele); sinais vitais e Doenças Crônicas Não - Transmissíveis (DCNT) relatadas, descrição do grau de consciência, relato de violência; queixa principal e consequência da queda (localização da fratura e lesões). Foram excluídos do estudo: indivíduo idoso (mencionado no boletim) que não constasse a idade, o sexo, o dia e o local da ocorrência/atendimento, bem como registro incompleto ou ausente no boletim do motivo do atendimento; indivíduo idoso cuja procedência fosse de municípios de outras regiões do Estado do Rio Grande do Sul que não da Região Metropolitana.

Neste estudo usamos a classificação das consequências da queda de Caberlon e Bós (2015) ${ }^{(14)}$. Nesta classificação as informações sobre fratura, presença de escoriação, edema, equimose e dor são classificadas conforme a gravidade das lesões apresentadas. Diferentes lesões recebem notas diversas conforme a gravidade atribuída. As fraturas recebem nota 10, escoriações 5 , equimose 4 e edema e dor 1 . Desta forma uma pessoa que teve uma fratura (10), uma escoriação (5) e uma equimose (4) receberam gravidade 19. Os níveis de gravidade foram posteriormente classificados conforme esta distribuição: zero pontos (sem gravidade), 1 ponto (gravidade muito leve), 2 a 4 pontos (leve), 5 a 9 pontos (moderada), 10 a 14 pontos (severa) e 15 ou mais pontos (muito severa).

As médias da gravidade da queda foram comparadas entre os dois grupos etários e testada pelo teste $t$ de Student. A relação entre o gênero, faixa etária e o grau de gravidade foi testado pelo qui-quadrado. $\mathrm{O}$ teste não paramétrico de Kruskal-Wallis foi aplicado observando relação significativa entre faixa etária e os resultados dos valores da gravidade da queda. Foi considerado estatisticamente significativo p menor que 5\%. Os dados foram analisados no programa Epi Info тм 7.015. O estudo foi aprovado pelo Comitê de Ética em Pesquisa da PUCRS, sob o protocolo 09/04881 e pelas instituições hospitalares sob os protocolos 562 (Hospital de Pronto Socorro) e 11/049 (Hospital Cristo Redentor). Os autores declaram não haver conflito de interesses no presente trabalho.

\section{RESULTADOS}

No período de $1^{\mathrm{o}}$ de janeiro a 31 de dezembro de 2010, foram atendidos 4681 pessoas com 60 anos e mais, pelo motivo queda, nas duas unidades de UAUEH estudadas. Desses 3636 (77.78\%) eram idosos jovens e 1045 (22,32\%) eram longevos. A maioria dos atendidos era do sexo feminino $(72,10 \%)$, entre as quais $23,7 \%$ eram longevas. A percentagem de longevas foi significativamente maior que o de longevos, com 18,76\% (p<0,001) (Tabela 1).

Tabela 1. Distribuição dos idosos atendidos por queda segundo sexo e faixa etária.

\begin{tabular}{lccc}
\hline Faixa etária & Feminino & Masculino & Total* \\
Idosos & $2575(70,82 \%)$ & $1061(29,18 \%)$ & $3636(77,68 \%)$ \\
Longevos & $800(76,56 \%)$ & $245(23,44 \%)$ & $1045(22,32 \%)$ \\
Total & $3375(72,10 \%)$ & $1306(27,90 \%)$ & $4681(100,00 \%)$ \\
\hline
\end{tabular}

$* p<0,001$.

A média do índice de gravidade geral foi de 5,7. Longevos apresentaram um índice de 6,5282 $\pm 5,4506$ enquanto os idosos mais jovens apresentaram um índice menor de 5,4807 $\pm 6,3921$. As medianas da gravidade da queda também se apresentaram maiores nos longevos, observando que a maioria dos longevos apresentaram pior gravidade da queda do que os idosos jovens, apesar do idoso com maior gravidade ter tido uma queda com consequências mais severas que o longevo com pior queda. As diferenças entre a mediana e a média da queda falam a favor da sua distribuição não ser homogênea, indicando que a análise não paramétrica seja a mais adequada para a variável dependente (gravidade da queda). O teste não paramétrico de Kruskal-Wallis foi aplicado observando relação significativa entre faixa etária e os resultados dos valores da gravidade da queda $(\mathrm{p}<0,001)$ (Tabela 2). 
Tabela 2. Distribuição dos idosos atendidos por queda segundo escore de gravidade e por faixa etária.

\begin{tabular}{lcccc}
\hline Faixa etária & Total* & Média & Desvio & Mediana \\
Idosos & 3636 & 5,4807 & 6,3921 & 30000 \\
Longevos & 1045 & 6,5282 & 5,4506 & 50000 \\
\hline
\end{tabular}

$* p<0,001$.

Neste estudo, observou-se que as mulheres apresentaram maior frequência de quedas. Da mesma forma, o grau de gravidade severa mostrou-se ser maior nelas. Assim, tal fato nos leva a crer que as mulheres fraturam mais quando caem. Observou-se que longevos têm quedas mais frequentes que idosos jovens com gravidade moderada e severa independente do sexo. Observou-se também que quanto maior a gravidade da queda maior é a frequência de longevos. Queda sem gravidade mostra-se maior em homens no geral e nos idosos jovens independente do sexo. A diferença percentual de quedas sem gravidade é maior entre idosos jovens e longevos do sexo masculino, pois a diferença é menor nas mulheres. Já as quedas severas foram mais frequentes nas mulheres em geral e nos longevos independente do sexo (Tabela 3 ).

\section{DISCUSSÃO}

No presente estudo, observou-se que a maior parte dos indivíduos idosos atendidos por queda era do sexo feminino, entre as quais observa-se um maior percentual de longevas. Um estudo internacional, ao comparar a ocorrência de quedas entre os gêneros, encontrou nas mulheres uma probabilidade de risco para quedas de 1,94 vezes maior do que em homens ${ }^{16}$. Acredita-se que tal prevalência possa estar relacionada ao melhor estado funcional e maior mobilidade das mulheres idosas, com maior uso de medicamentos em comparação aos homens idosos, favorecendo assim, maior exposição ao risco de queda ${ }^{17}$. $\mathrm{O}$ fato das mulheres terem envolvimento maior com atividades domésticas parece ser outro fator que as deixaria mais expostas a tal evento ${ }^{18}$. Por outro lado, para Rodrigues et al. (2014) ${ }^{19} \mathrm{o}$ fato das mulheres sofrerem mais quedas pode estar relacionado à menor quantidade de massa magra e de força muscular que elas apresentam em relação aos homens da mesma idade.

Além da prevalência observada no público feminino, a idade avançada parece ser outro fator predisponente para a queda. Para Ferreira e Yoshitome $(2010)^{20} \mathrm{com}$ o passar da idade, o corpo sofre alterações fisiológicas que favorecem o acontecimento das quedas, como declínio da força muscular, alterações na massa óssea, déficit de equilíbrio, diminuição dos reflexos, diminuição da coordenação motora, diminuição da flexibilidade, além de deficiências visuais, deficiências de propriocepção e deficiências do sistema vestibular. Além da frequência de quedas, as mulheres apresentaram maior gravidade severa em relação aos homens, indicando que elas fraturam mais quando caem. Cruz et al. $(2012)^{21} \mathrm{em}$ seu estudo observaram que a presença de fraturas após uma queda está diretamente relacionada a presença de osteoporose, ao sexo feminino e à idade avançada. Tal fato nos leva a crer que as mulheres fraturaram mais por serem mais acometidas pela osteoporose do que homens, embora não tenhamos analisado a presença de osteoporose nos participantes deste estudo.

Tabela 3. Distribuição dos idosos atendidos por queda segundo sexo, faixa etária e por grau de gravidade.

\begin{tabular}{|c|c|c|c|c|c|c|}
\hline & Sem gravidade & Leve & Moderada & Severa & Total & Valor $p$ \\
\hline \multicolumn{7}{|l|}{ Sexo } \\
\hline Feminino & $523(15,50 \%)$ & $1189(35,23 \%)$ & $534(15,82 \%)$ & $1129(33,45 \%)$ & $3375(72,1 \%)$ & \multirow[t]{2}{*}{$<0,001$} \\
\hline Masculino & $215(16,46 \%)$ & $369(28,25 \%)$ & $313(23,97 \%)$ & $409(31,32 \%)$ & $1306(27,90 \%)$ & \\
\hline \multicolumn{7}{|l|}{ Faixa etária } \\
\hline Idosos & $592(16,28 \%)$ & $1328(36,52 \%)$ & $589(16,20 \%)$ & $1127(31,00 \%)$ & $3636(77,68 \%)$ & \multirow[t]{2}{*}{$<0,001$} \\
\hline Longevos & $146(13,97 \%)$ & $230(22,01 \%)$ & $258(24,69 \%)$ & $411(39,33 \%)$ & $1045(22,32 \%)$ & \\
\hline \multicolumn{7}{|l|}{ Mulheres } \\
\hline Idosos & $409(15,88 \%)$ & $1001(38,87 \%)$ & $353(13,71 \%)$ & $812(31,53 \%)$ & $2575(76,30 \%)$ & \multirow[t]{2}{*}{$<0,001$} \\
\hline Longevos & $114(14,25 \%)$ & $188(23,50 \%)$ & $181(22,63 \%)$ & $317(39,63 \%)$ & $800(23,70 \%)$ & \\
\hline \multicolumn{7}{|l|}{ Homens } \\
\hline Idosos & $183(17,25 \%)$ & $327(30,82 \%)$ & $236(22,24 \%)$ & $315(29,69 \%)$ & $1061(81,24 \%)$ & \multirow[t]{2}{*}{$<0,001$} \\
\hline Longevos & $32(13,06 \%)$ & $42(1714 \%)$ & $77(31,43 \%)$ & $94(38,37 \%)$ & $245(18,76 \%)$ & \\
\hline
\end{tabular}


De acordo com a classificação das consequências da queda proposta por Caberlon e Bós (2015) ${ }^{15}$ constatamos que o índice de gravidade da queda é maior em longevos. Para Lourenço et al. (2012) ${ }^{22}$ este achado pode ser justificado pela própria senescência que causa diminuição da massa corporal e óssea, interferindo na capacidade funcional, podendo assim causar maior número de quedas com maior gravidade. Outro aspecto relevante é o fato dos longevos apresentarem maior frequência de doenças crônicas, maior tendência ao sedentarismo, maior tendência ao uso de medicamentos, assim como alterações de mobilidade que acabam por interferir na realização das Atividades de Vida Diária (AVD) favorecendo as quedas ${ }^{23}$. Soldera et al. (2015) ${ }^{24}$ observou pior desempenho em todos os sistemas de manutenção do equilíbrio corporal em longevos quando comparados a idosos mais jovens.

Os longevos apresentaram maior frequência de quedas e aumento gradual da gravidade das quedas, com predomínio de moderada e severa, em comparação a idosos mais jovens. Tal fato corrobora com o estudo de Rodrigues et al. (2014) ${ }^{19}$ realizado com idosos não institucionalizados, residentes em área urbana do município de Campinas, São Paulo, que apontou a ocorrência de quedas 2,5 vezes maior em longevos quando em comparação com idosos jovens. Esse resultado é atribuído às alterações que ocorrem com o processo de envelhecimento, como perdas progressivas de equilíbrio e alterações na massa muscular e óssea.

Em contrapartida, a queda sem gravidade mostrase maior em homens e nos idosos jovens. Tal achado parece ser explicado pelo fato de que os idosos homens, assim como os jovens, tendem a se expor menos do que as mulheres, que consequentemente se mantêm mais ativas, tanto no lar quanto socialmente, favorecendo assim menor número de quedas e consequentemente menor gravidade. Um fato que corrobora esta hipótese é o observado no estudo de Caberlon e Bós (2015) ${ }^{15}$ em que de um total de 173 idosos atendidos em UAUEH devido queda dentro do ônibus, 139 (80,3\%) eram mulheres. A Pesquisa do Perfil dos Idosos do Rio Grande do Sul de 2010 observou que 34,7\% dos idosos gaúchos dirigem carro contra apenas 9,2\% das idosas ${ }^{25}$.

Para o entendimento e a discussão dos resultados obtidos no presente estudo apontamos algumas limitações. O presente estudo foi realizado através da observação das informações contidas nos boletins de atendimento. Foi possível observar a falta de informações importantes sobre as circunstâncias da queda (local e horário da queda), assim como a ausência da observação sistemática do estado de consciência do paciente no momento do atendimento nos boletins. $O$ índice de gravidade da queda proposto neste artigo não leva em consideração o local de cada lesão isoladamente o que poderia ter interferido diretamente na gravidade da queda. Uma análise futura será possível de ser realizada e observar se a inclusão do local da lesão produziria um índice mais confiável.

\section{CONCLUSÃO}

Concluí-se que idosos longevos apresentam maior gravidade das quedas. Essa conclusão também é válida para o sexo feminino que, além de caírem com maior frequência apresentam maior gravidade das quedas principalmente no número de fraturas nas faixas etárias avaliadas (idosos jovens e longevos). Concluise também ser possível utilizar um instrumento de avaliação do grau da queda que poderá ser utilizada em investigações futuras. Ressaltamos a importância deste estudo no que tange a qualificação de um evento extremamente preocupante para a população idosa, ao passo que buscamos uma investigação direta aonde essa demanda mostra-se evidente, nos serviços de emergência. Tal fato nos leva a crer, a necessidade dessa investigação, principalmente através de escalas que estratifiquem de fato a gravidade da queda, a fim de contribuir para a realização de uma vigilância epidemiológica mais eficaz e resolutiva.

\section{CONFLITO DE INTERESSE}

Os autores do presente estudo declaram a inexistência de conflito de interesses.

\section{REFERÊNCIAS}

1. Minayo, MCS. O envelhecimento da população brasileira e os desafios para o setor saúde Cadernos de Saúde Pública 2012; 28(2):208-9.

2. INSTITUTO BRASILEIRO DE GEOGRAFIA E ESTATÍSTICA (IBGE. Censo de 2010. 2010 [acesso em 12 jul. 2016]. Disponível em: http://www.ibge.gov.br/home/ estatistica/populacao/censo2010/ default.shtm

3. Küchemann BA. Envelhecimento populacional, cuidado e cidadania: velhos dilemas e novos desafios. Revista Sociedade e Estado 2012; 27 (1):165-80.

4. INSTITUTO BRASILEIRO DE GEOGRAFIA E ESTATÍSTICA (IBGE). Censo de 2000, 2000. Disponível em: http://www.ibge.gov.br/home/estatistica/populacao/ censo2000/. Acesso em: 12 jul. 2016.

5. Esquenazi D, Silva SRB, Guimarães, MAM. Aspectos fisiopatológicos do envelhecimento humano e quedas em idosos. Revista HUPE 2014; 13(2):11-20.

6. Giro A, Paúl C. Envelhecimento Sensorial, Declínio Cognitivo e Qualidade de Vida no Idoso com Demência. Actas de Gerontologia 2013; 1(1):1-10. 
7. Landi F, Liperoti R, Russo A, Capoluongo E, Barillaro C, Pahor $\mathrm{M}$, et al. Disability, more than multimorbidity, was predictive of mortality among older person aged 80 years older. Journal of Clinical Epidemiology. 2010;63(7):752-9.

8. Nogueira SL, Ribeiro RCL, Rosado LEFPL, Franceschini SCC, Ribeiro AQ, Pereira ET. Fatores determinantes da capacidade funcional em idosos longevos. Revista Brasileira de Fisioterapia. 2010;14(4):322-9.

9. Pinho TAM, Silva AO, Tura LFR, Moreira MASP, Gurgel SN, Smith AAF. Avaliação do risco de quedas em idosos atendidos em Unidade Básica de Saúde. Revista da Escola de Enfermagem da USP. 2012;46(2):320-7.

10. Brito TA, Fernandes MH, Coqueiro RS, Jesus CS. Quedas e capacidade funcional em longevos residentes em comunidade. Texto e Contexto Enfermagem. 2013;22(1): 43-51.

11. Maia BC, Viana PS, Arantes PMM, Alencar MA. Consequências das quedas em idosos vivendo na comunidade. Revista Brasileira de Geriatria e Gerontologia. 2011;14(2):381-93.

12. Moraes EM, Marino MCA, Santos RR. Principais síndromes geriátricas. Revista Médica de Minas Gerais. 2010;20(1): 54-66.

13. Cunha AA, Lourenço RA. Quedas em idosos: prevalência e fatores associados. Revista HUPE. 2014;13(2):21-9.

14. Caberlon IC, Bós AJG. Diferenças sazonais de quedas e fraturas em idosos gaúchos. Ciência \& Saúde Coletiva. 2015; $20(12): 3743-52$.

15. Bós AJG. Epi Info ${ }^{\circledR}$ sem Mistérios: Um manual prático. Porto Alegre: EDIPUCRS; 2012.

16. Lin CH, Liao KC, Pu SJ, Chen YC, Liu MS. Associated factors for falls among the community-dwelling older people assessed by annual geriatric health examinations. PLoS One. $2011 ; 6(4): 1-5$.

17. Lopes RA, Dias RC. O impacto das quedas na qualidade de vida dos idosos. Revista ConScientie Saúde. 2010;9(3): 504-9.

18. Álvares LM, Lima RC, Silva, RA. Ocorrência de quedas em idosos residentes em instituições de longa permanência em Pelotas, Rio Grande do Sul, Brasil. Cadernos de Saúde Pública. 2010;26(1):31-40.

19. Rodrigues IG, Fraga GP, Barros MBA. Quedas em idosos: fatores associados em estudo de base populacional. Revista Brasileira de Epidemiologia. 2014;17(3):705-18.

20. Ferreira DCO, Yoshitome AY. Prevalência e características das quedas de idosos institucionalizados. Revista Brasileira de Enfermagem. 2010;63(6):991-7.

21. Cruz DT, Ribeiro LC, Vieira MT, Teixeira BTB, Bastos RR, Leite ICG. Prevalência de quedas e fatores associados em idosos. Revista de Saúde Pública. 2012;46(1):138-46.

22. Lourenço TM, Lenardt MH, Kletemberg DF, Seima MD, Tallmann AEC, Neu DKM. Capacidade funcional no idoso longevo: uma revisão integrativa. Revista Gaúcha de Enfermagem. 2012;33(2):176-85.

23. Oliveira BS. Relação entre capacidade funcional e sinais de violência e maus tratos em idosos longevos. Fisioterapia Brasil. 2015;16(1):32-7.

24. Soldera CLC, Oliveira GG, Bós AJG. Differences in Dynamic Posturography Results Between Older-Adult and OldestOld. Clinical Medicine Journal. 2015;1(4):115-21.

25. Morsch P, Ribeiro A. Características do idoso gaúcho e do seu ambiente. In: Bós AJG, Mirandola AR, Lewandowski A, Schirmer CL. Perfil dos idosos do Rio Grande do Sul. Porto Alegre: Escola de Saúde Pública; 2015. 356 p. 